\title{
L'art de la négociation: emmener la chèvre en passant
}

\section{Werner Bauer}

Dr med., membre de la rédaction

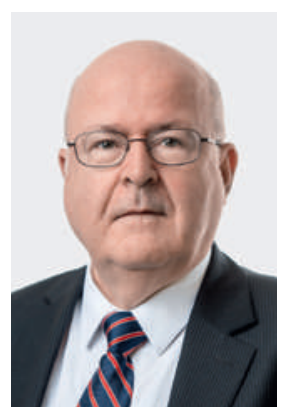

Négocier fait aujourd'hui partie des tâches quotidiennes de toute personne investie de responsabilités, quel que soit son secteur d'activité. Dans le domaine de la santé comme ailleurs, les (bien trop) longs colloques font partie du quotidien. Qu'il s'agisse de négociations internes d'un hôpital, de négociations de représentants du corps médical avec des assureurs, des organismes d'Etat ou d'autres institutions, de discussions entre médecins ou avec d'autres professionnels de la santé, il y est question de postes, de projets, d'honoraires, de financement, de régulation, de structures ou de directives. Nous vivons un intense ballet de négociations en vue de la révision du tarif médical ambulatoire. Les médecins sont-ils des négociateurs compétents parce qu'être médecin leur donne certaines compétences?

J'ai encore tout juste connu l'époque où, par exemple, le comité d'une caisse-maladie de district tenait ses séances à la fin d'une journée de travail, le délégué du corps médical n'arrivant qu'après sa dernière visite à domicile. Les représentants de la caisse étaient des personnalités connues de la région, comme le buraliste postal ou l'administrateur de la caisse d'épargne locale. Tous officiaient à titre accessoire, portés sur la recherche d'un consensus, et les représentants des médecins, forts de leur savoir, de leur expérience à faire valoir leurs arguments et de leur position dans la commune et la région, n’avaient généralement aucune peine à défendre efficacement leurs principales revendications et à influencer les décisions en ce sens.

Les temps ont changé. Les petites caisses-maladie ont été fondues dans de grandes compagnies, les séances ont lieu pendant les heures de travail et les interlocuteurs des médecins ne sont plus des buralistes ou directeurs de caisse d'épargne bien disposés à leur égard, mais des collaborateurs qui raisonnent d'abord en termes économiques, ont une tactique rodée et sont tenus de défendre les intérêts de leur employeur. Il en va de même pour les parties négociant avec les médecins dans les hôpitaux, les organismes d'Etat et bien d'autres organisations.

La technique de négociation rejoint ainsi les disciplines dont la maîtrise est décisive pour assumer avec succès des mandats médicaux. Il existe une littérature et une offre de cours abondantes à ce sujet. Un site web consacré (www.rhetorik.ch) définit les éléments de base es- sentiels à la négociation: solide connaissance du dossier (donc une préparation détaillée et non un survol des documents dans le train avant la réunion), sens logique, force de persuasion et compréhension psychologique. «Gagner sans triompher» désigne un objectif de négociation permettant aux deux parties de conclure une négociation avec un sentiment positif, condition nécessaire d'une solution durable vraiment acceptée.

A propos de l'art de la négociation, je me souviens d'une conférence et de deux livres de Harro von Senger, qui était professeur de sinologie à Fribourg-en-Brisgau: 36 Strategeme für Manager [1] et Die Kunst der List. Strategeme durchschauen und anwenden [2]. Le mot «stratagème» est souvent compris dans le sens de «ruse», mais les stratagèmes sont le reflet d'une technique de ruse au sens positif du terme, basée sur l'intelligence et l'ingéniosité bien plus que sur l'astuce et la tromperie. Alors que le philosophe des Lumières anglais John Locke voyait dans la ruse le «singe de la sagesse», pour les Chinois, la sagesse et l'intellect sont les sources dont elle se nourrit. Un stratagème utilisé au bon moment peut être décisif pour infléchir le cours d'une négociation ou d'une action dans le sens souhaité. Connaître les stratagèmes peut aider les deux parties à mieux percevoir les manipulations et à trouver une solution acceptable. Il existe différentes catégories de stratagèmes (pour une étude approfondie, je renvoie aux publications citées de Harro von Senger) que chacun peut utiliser selon la force de sa position et l'état d'avancement des négociations.

- Décorer de fleurs un arbre sec (stratagème du bluff).

- Attirer le tigre hors de sa montagne (stratagème de l'isolement).

- Maudire l'acacia en désignant le mûrier (stratagème de la critique indirecte).

- Emmener la chèvre en passant (rester à l'affût des chances qui se présentent).

- Relâcher prise pour mieux saisir (vision à long terme). - Regarder le feu depuis l'autre rive (se tenir à l'écart). $\mathrm{Au}$-delà des problèmes à résoudre, les facteurs décisifs de succès seront toujours la technique et l'art de la négociation - y compris pour le médecin: aucun tigre attiré hors de sa montagne ne doit pouvoir profiter d'être caché par un arbre sec décoré de fleurs pour emmener la chèvre en passant! 